\title{
ANÁLISIS COMPARATIVO DE MODELOS DE EVALUACIÓN DE CALIDAD EN EL SERVICIO A PARTIR DE SUS DIMENSIONES Y SU RELACIÓN CON LA SATISFACCIÓN DEL CLIENTE
}

\section{COMPARATIVE ANALYSIS OF SERVICE QUALITY EVALUATION MODELS BASED ON THEIR DIMENSIONS AND THEIR RELATIONSHIP WITH CUSTOMER SATISFACTION}

1. Licenciada, Universidad Autónoma de Baja California Sur (México). E-mail: Lluvianunez90@gmail.com

2. Doctora, Universidad Autónoma de Baja California Sur (México). E-mail: Judithjuarezm@gmail.com

\section{Citación sugerida:}

Núñez Tobías, L.N. y Juárez Mancilla, J. (2018). Análisis comparativo de modelos de evaluación de calidad en el servicio a partir de sus dimensiones y su relación con la satisfacción del cliente. $3 C$ Empresa, investigación y pensamiento crítico, 7(1), 49-59. DOI: <http://dx.doi.org/10.17993/3cemp.2018.070133.49-59/>. 


\section{RESUMEN}

En este trabajo se estudian los factores que intervienen en la valoración llevada a cabo por el consumidor una vez que experimentó el servicio, y cómo contribuyen a explicar el nivel de calidad en el servicio que el cliente asigna a la experiencia ha propiciado el surgimiento de modelos de evaluación de calidad en el servicio. Se estudian las dimensiones propuestas en 8 modelos de evaluación de calidad en el servicio que son reconocidos y aceptados en el área analizando la dirección de la relación causal de los factores identificados en cada uno de los modelos hacia el constructo satisfacción del cliente. Estableciendo una categorización de determinantes de la calidad se identificó la dimensión que evoca al personal de contacto como la de mayor presencia en los modelos de evaluación de calidad en el servicio analizados, al igual que los elementos tangibles presentes en la experiencia de consumo.

\section{ABSTRACT}

In this paper are studied the factors involved in the assessment carried out by the consumer once the service was experienced, and how that contributes to explain the level of quality in the service that the client assigns to the experience has led to the emergence of quality of service evaluation models. It is studied the dimensions proposed in 8 quality models that are recognized and accepted in the area by analyzing the direction of the causal relationship of the factors identified in each of the models towards the customer satisfaction construct. By establishing a categorization of quality determinants it was identified the dimension that evokes the contact staff as the one with the greatest presence in the quality evaluation models in the service analyzed, as well as the tangible elements present in the consumer experience.

\section{PALABRAS CLAVE}

Calidad en el servicio, satisfacción del cliente, SERVQUAL, Dimensiones de calidad.

\section{KEY WORDS}

Quality service, Customer satisfaction, SERVQUAL, Quality dimensions. 
Fue a partir del surgimiento de la gestión de calidad en los años $80^{\prime}$ como hoy la conocemos, que se dejó atrás la visión de la calidad como el mero cumplimiento de los requisitos (Pérez, 2014), reconociéndola como una orientación a la mejora continua del rendimiento a largo plazo de una organización (Diamandescu, 2016). Es entonces cuando surgen modelos de evaluación de la calidad en el servicio, cuyo objetivo es medir el nivel de calidad valorado por los clientes al momento de experimentar el servicio, logrando identificar áreas de oportunidad en las que la empresa debe enfocarse con mayor premura para que el consumidor describa su servicio como de alta calidad o, según algunos autores, como satisfactorio. Los factores, dimensiones o componentes que deben ser susceptibles a evaluación, a través de los cuales se logra explicar y, por lo tanto, medir el nivel de calidad en el servicio percibido por los consumidores inicia la discusión. El presente trabajo se realizó con un enfoque cualitativo con base en la revisión de la literatura a partir de distintas bases de datos a través de la técnica de triangulación teórica y utilizando el software Nvivo para organizar, analizar y encontrar coincidencias, se lograron identificar los factores, dimensiones o componentes que distintos autores consideran como explicativos de esta calidad; con esto se realizó un análisis comparativo buscando determinar las similitudes entre las teorías examinadas, así como el factor de más frecuencia.

\section{CALIDAD EN EL SERVICIO}

Vista desde la perspectiva organizacional, la calidad en el servicio es la orientación que siguen todos los recursos de una empresa para lograr la satisfacción de los clientes incluyendo a todos los empleados y no solo a los que tienen trato directo con el cliente (Kotler \& Keller, 2012), pues todas las actividades realizadas por un empleado en la organización repercutirán de alguna manera en el nivel de calidad real o percibida por el consumidor. A partir de esto se construye la reputación, que será buena si el servicio se percibe como de alta calidad, dando como resultado una mayor repetición en las ventas (Tschohl, 2011). La calidad puede abordarse específicamente desde la perspectiva de la conformidad de las especificaciones, la satisfacción de las expectativas del cliente, la excelencia de un producto o servicio o, como es actualmente manejado, un concepto global e integrador que involucra a toda la organización (Camisón, Cruz, \& González, 2006). Sin embargo es la calidad desde el punto de vista del consumidor la que compete a este estudio, fue definida por Parasuraman, Zeithaml, y Berry en 1988 como "un juicio global, o actitud relacionada con la superioridad del servicio", tomando en consideración esto, algunos autores identificaron lo que se conoce como el paradigma de la disconfirmación, que en palabras simples es la discrepancia entre expectativas y percepciones de la calidad del servicio (Oliver, 1977; Grönroos, 1984; Parasuraman, Zeithaml, \& Berry, 1988). 


\section{SATISFACCIÓN DEL CLIENTE}

La satisfacción del cliente es definida como "el grado en el que el desempeño percibido de un producto coincide con las expectativas del consumidor" (Kotler \& Armstrong, 2012), y aguarda una gran similitud con la definición que se dio del paradigma de la disconfirmación, por lo que la descripción dada por Oliver en 1981 parece aún más adecuada para este estudio: "la satisfacción del cliente es el estado psicológico que resulta cuando la emoción que rodea las expectativas disconfirmadas está a la par de los sentimientos previos del consumidor sobre la experiencia de consumo". En primera instancia debemos precisar que el cliente y el consumidor pueden o no ser la misma persona, la disparidad radica en que es el primero quien paga por el producto o servicio que vende la organización, mientras que el segundo es quien hace uso de este bien o servicio (Ayala, Camino, \& Cueva, 2013). Un cliente generalmente es también un consumidor, pero un consumidor no es necesariamente un cliente. Desde el enfoque cognitivo, se entiende la satisfacción como un proceso donde los sujetos analizan racionalmente la situación compra/venta, generando posteriormente la satisfacción o insatisfacción (Baena, García, Bernal, Lara, \& Gálvez, 2016). En el enfoque afectivo del concepto de satisfacción, los resultados obtenidos por el consumidor durante la experiencia de consumo provocan dos tipos de evaluaciones, primero se genera una evaluación inicial en términos de éxito o fracaso que induce un estado afectivo originando agrado o desagrado en el consumidor, y segundo se genera una evaluación secundaria que se refiere a las diferentes emociones (que pueden ser enfado, gratitud, culpabilidad, frustración, enfado, arrepentimiento) que se forman después de un proceso de atribución casual (Moliner, Gil, \& Ruíz, 2014). A partir de esto podemos decir que la satisfacción del cliente es una respuesta emocional resultante de una experiencia de consumo, que en el caso de los servicios se da durante y después del mismo. Por otro lado, los conceptos son construcciones teóricas separables que ocupan posiciones únicas en una red nomológica determinada por conjuntos únicos de causas-antecedentes, efectos consiguientes o ambos (Chandrasegaran, y otros, 2013), pero si dos conceptos de red comparten todos los antecedentes y consecuencias teóricas entonces son "estructuralmente equivalentes" o lógicamente isomórficos, y discutirlos como si fueran únicos sería indefendible y no comprobable empíricamente. En este caso las posiciones de calidad y satisfacción en la red nomológica no son únicas, sino estructuralmente intercambiables (Berkowitz, 2013). Por lo que según los autores se requiere identificar otros factores que causan o afectan diferencialmente la calidad y la satisfacción que deben ser conceptualizados y probados si se quiere distinguir entre ambos, y es en esos factores explicativos de la calidad en los cuales se enfoca el presente trabajo. 


\section{MODELOS PARA LA EVALUACIÓN DE LA CALIDAD EN EL SERVICIO}

Los atributos, dimensiones o factores que, según los distintos modelos, definen el nivel de calidad en el servicio de una empresa a través de la percepción de sus clientes continua siendo tema de discusión entre los autores debido a la subjetividad que representa la calidad percibida y los innumerables aspectos que un individuo puede tomar en cuenta al momento de valorarla. Además, es posible identificar los modelos que tratan la satisfacción del cliente como una consecuencia de la calidad en el servicio, y los que, contrariamente, explican la calidad en el servicio a partir de la satisfacción. A continuación se exponen algunos de los modelos que hablan de la calidad en el servicio como un antecedente de la satisfacción.

Christian Grönroos en 1984 explicó la calidad percibida de un servicio como el resultado de un proceso de evaluación donde el consumidor compara su expectativa con el servicio que percibe haber recibido. Se trata de un modelo de tres componentes: la calidad técnica, la calidad funcional y la imagen corporativa. Grönroos se refiere a la dimensión de la calidad técnica cuando habla de lo que el cliente recibe como resultado técnico del proceso de producción, y esta responde a la pregunta de ¿qué consigue?; mientras que la dimensión de la calidad funcional corresponde al desempeño expresivo de un servicio, es decir al ¿cómo el cliente consigue el servicio?, lo que es percibido de manera subjetiva. Se puede esperar que la imagen corporativa se construya principalmente por la calidad técnica y la calidad funcional de sus servicios, además pueden influir también en la imagen otras variables: factores externos y actividades tradicionales de marketing.

Parasuraman, Zeithaml y Berry en 1985 desarrollaron el modelo de calidad en el servicio denominado SERVQUAL. Identificaron 10 criterios de evaluación que llamaron "determinantes de la calidad en el servicio", siendo a raíz de las críticas y la verificación empírica que el modelo fue actualizado por los autores quedando simplificado a una evaluación de 5 dimensiones: empatía, se refiere a la ofrecida por la empresa a sus clientes; fiabilidad: la habilidad de ejecutar el servicio prometido; seguridad, definida como la confianza y credibilidad que inspiran los empleados según sus conocimientos y atenciones a los clientes; capacidad de respuesta: rápida disposición para ayudar y prestar el servicio; por último, los elementos tangibles que es la apariencia física de las instalaciones, personal y materiales (Parasuraman, Zeithaml, \& Berry, 1988). El modelo está fundamentado en la teoría de la disconfirmación, de manera que cuando se cumple exactamente el nivel de expectativas del usuario se define el servicio como satisfactorio.

Posteriormente, J. Cronin y S. Taylor en 1992 sugieren que la conceptualización y operacionalización de la calidad de servicio (SERVQUAL) es inadecuada, sustentándose en que, en ese momento, poca o ninguna evidencia teórica o empírica apoyaba la relevancia de las expectativas-rendimiento como base para medir la calidad del servicio. Sin embargo, coinciden con Parasuraman, Zeithaml, and Berry $(1985,1988)$ al afirmar que la calidad del 
servicio percibido conduce a la satisfacción del consumidor. Los autores establecieron una escala más concisa a la que llamaron SERVPERF, basándola únicamente en la valoración de las percepciones de los mismos 5 factores que propone el modelo SERVQUAL.

El modelo de los tres componentes expresado por Rust \& Oliver (1994) establece que las percepciones de calidad no requieren experiencia con el servicio o proveedor; mientras que la satisfacción es puramente experiencial. Los autores delimitan a tres componentes siempre presentes en la calidad en el servicio: el servicio y sus características, el ambiente del servicio y el servicio entregado. El primero se refiere a cómo el servicio fue diseñado para ser entregado, incluyendo sus características específicas y objetivos del servicio. El ambiente del servicio se dividió en interno: cultura organizacional y filosofía de la dirección para proveer el servicio, y externo: ambiente físico del servicio.

En contraste, a continuación se describen y analizan modelos de evaluación de calidad en el servicio donde la satisfacción influye o determina la calidad en el servicio percibido.

Bitner (1990) en su propuesta afirma que la reacción inmediata del cliente después del consumo depende de una comparación de las expectativas anteriores y el desempeño percibido, lo que da como resultado la confirmación de las expectativas cuando no coinciden con el desempeño. Se trata de un modelo que plantea un proceso en etapas, y como primera entrada sugiere el marketing mix ampliado, es decir, los cuatro elementos tradicionales (producto, precio, lugar, promoción) y tres nuevos (evidencia física, participantes, proceso). El autor utilizó la técnica del incidente crítico para descubrir las fuentes subyacentes de satisfacción e insatisfacción en los encuentros de servicio. Bitner, Booms, \& Stanfield (1990) definieron los incidentes críticos como interacciones específicas entre clientes y empleados de la empresa de servicios que podrían explicar todos los incidentes satisfactorios e insatisfactorios. El marketing mix entra en el proceso de satisfacción del encuentro de servicio influyendo directamente en tres antecedentes de satisfacción: las expectativas, el desempeño del servicio percibido y las atribuciones. Según el autor, no es la calidad del servicio o el fracaso de atender una necesidad o petición especial lo que causa insatisfacción sino más bien el carácter evaluado o la actitud del empleado.

Para realizar la evaluación, Bolton y Drew (1991) definieron la calidad en el servicio como una actitud frente a la empresa y todos sus servicios; utilizan el paradigma de la disconfirmación para explicar la calidad proponiendo un modelo multietapa que hace una evaluación global del servicio al descomponerse en una serie de etapas interrelacionadas: evaluaciones de rendimiento, calidad de servicio y valor. En la evaluación del rendimiento encuentran como variables explicativas la fiabilidad, la capacidad de respuesta, la seguridad y las dimensiones de la empatía, además de las experiencias pasadas en diferentes empresas. La evaluación del cliente, según los autores, se basa en su satisfacción/insatisfacción con el servicio actual, que depende del desempeño percibido de los componentes del servicio, las expectativas previas sobre el desempeño y las percepciones de la discrepancia entre rendimiento y expectativas. El valor del servicio está 
dado por el sacrificio relativo de los clientes en su presupuesto, sus características e intenciones. El modelo acepta que un cliente puede sopesar los componentes subyacentes de la calidad del servicio de manera diferente en la evaluación del valor del servicio. Por lo tanto la satisfacción es un antecedente de la calidad en el servicio, que le asignará un valor al servicio que a su vez derivará en intenciones de compra.

Nha Nguyen en 1991 propuso un modelo inspirado en el sistema de servucción, lo que se refiere a la organización sistemática y coherente de todos los elementos físicos y humanos de la relación cliente-empresa necesaria para la realización de una prestación de servicio cuyas características comerciales y niveles de calidad han sido determinadas (Eiglier \& Langeard, 1987). Reconoció cinco factores: la imagen de la empresa: el personal de contacto, el medio físico, la organización interna del sistema de prestación de servicios y la satisfacción del cliente. La imagen de una empresa es por lo general influenciada por la tradición, ideología y cierta información transmitida por la propia empresa o por un tercero; a su vez la evaluación del personal en contacto se basa en su experiencia, actitud e incluso la demografía; mientras que el medio físico se define por el diseño y la disposición de los elementos tales como la ubicación, la construcción, interior y exterior, además de todos los objetos, muebles o máquinas utilizadas por el personal o cliente durante el sistema de prestación de servicios; la organización interna incluye, entre otras, los objetivos de la empresa, la estructura que adopta y las operaciones que lleva a cabo. De acuerdo con Nguyen la satisfacción es el resultado de una evaluación subjetiva instantánea y aplicable a la selección y el consumo del servicio.

Teas en 1993 argumentó que la satisfacción de transacciones es un predictor de la calidad percibida de la relación a largo plazo, afirmando que los atributos empleados en la medición de la calidad de servicio serán problemáticos según sean atributos vectoriales (atributos con puntos ideales infinitos) o atributos con puntos ideales finitos, para los que sugiere puntuaciones ponderadas; no plantea dimensiones en su modelo.

\section{DISCUSIÓN}

En su mayoría los modelos analizados valoran tanto expectativas como percepciones en el proceso de evaluación de calidad en el servicio llevado a cabo por el cliente. Para Grönroos (1984), Parasuraman, Zeithaml, y Berry (1985), Bitner (1990) y Bolton y Drew (1991) la empresa tiene que hacer coincidir el servicio esperado con el servicio percibido para lograr la satisfacción del consumidor. Sin embargo, es importante resaltar que no en todos los modelos analizados se utiliza el paradigma de la disconfirmación, tal es el caso del modelo del desempeño evaluado (Teas, 1993), ya que propone fijar una ponderación relativa a los aspectos a calificar adaptados a la empresa según el nivel de expectativa que se tenga de cada uno. Tanto Rust y Oliver (1994) como Cronin y Taylor (1992) coinciden en que la satisfacción es influenciada en gran medida por la calidad en el servicio percibida pero también dan crédito a otras variables. En la tabla 1 se categorizan la mayor parte de las 
determinantes de servicio mencionadas en los siguientes rubros: imagen organizacional, personal de contacto y lo que se refiere puramente a la organización del servicio. Así mismo se identificó cómo las dimensiones que tratan lo relacionado con el personal de contacto son más frecuentemente utilizadas por los teóricos para explicar la calidad en el servicio. Esto se sostiene en primer lugar por tres de las características distintivas de los servicios (Kotler \& Keller, 2012; Mercado, 2008) que establecen la estrecha relación entre un servicio y su prestador: inseparabilidad, la variabilidad y la especialidad. Los 8 modelos analizados consideran variables derivadas de la interacción del cliente con el personal para definir el nivel de calidad que ofrece una empresa de servicios pero, contrariamente, la imagen de la empresa no predomina como factor definitorio de la calidad; son Grönroos (1984) y Nguyen (1991) de la escuela europea, quienes determinan la imagen corporativa como una influencia directa. Sin embargo, los elementos físicos o tangibles se presentan con la misma frecuencia en los modelos estudiados que el personal en contacto, y esto se puede atribuir a la característica predominante de los servicios según Kotler y Keller (2012): intangibilidad, pues los clientes a menudo tienen problemas para evaluar la calidad del servicio en forma objetiva, y como resultado se basan en la evidencia física que rodea al servicio para ayudarlos a formar sus evaluaciones (Bitner, 1990; Hoffman \& Bateson, 2012). A partir del análisis realizado podemos afirmar que son los sistemas que intervienen en la realización del servicio y la armonía que estos guardan con los conocimientos y la actitud de los empleados de contacto lo que se refleja como constante en la evaluación de la calidad en el servicio.

\section{CONCLUSIONES}

El personal de contacto juega el papel principal en la evaluación consciente o inconsciente que el consumidor realiza del servicio recibido, aun así, es necesario reconocer los elementos físicos con los que el cliente interactúa como punto de partida para una primera evaluación inconsciente de la aptitud para servir, estos factores que dan una idea de la calidad ofertada son visibles hoy en día incluso antes de pisar el serviespacio, una página web, una aplicación móvil o red social pueden dar una imagen precisa de lo que le espera. El paradigma disconfirmatorio es frecuentemente aplicado, a pesar de los diversos métodos intentan disuadir la idea de que el sólo desajuste entre expectativas y percepciones arroje el valor de la calidad en el servicio. Sin embargo, la propuesta del modelo de desempeño evaluado que exige ponderar cada uno de los factores explicativos de la calidad tiene sustento, es aceptado y ampliamente utilizado. Siguiendo esta línea teórica podemos decir, para establecer una diferencia, que la satisfacción del cliente es o debería ser el fin de todas las empresas, al cual se llega a través de la calidad en el servicio. 
Tabla 1. Identificación de las dimensiones explicativas de la calidad en el servicio.

\begin{tabular}{|c|c|c|c|c|c|c|c|c|c|c|c|}
\hline & \multicolumn{4}{|c|}{ Antecedente } & \multicolumn{4}{|c|}{ Consecuencia } & \multicolumn{3}{|c|}{ Satisfacción } \\
\hline & $\begin{array}{l}\overrightarrow{0} \\
\text { 品 } \\
0\end{array}$ & $\begin{array}{l}\text { 范 } \\
0 \\
\end{array}$ & 䔎 & $\begin{array}{l}\text { 票 } \\
8\end{array}$ & 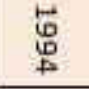 & $\begin{array}{l}\text { 莒 } \\
\text { N } \\
\end{array}$ & $\begin{array}{l}5 \\
5 \\
\infty \\
\infty \\
\infty\end{array}$ & 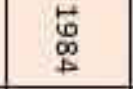 & ? & & \\
\hline & 营 & 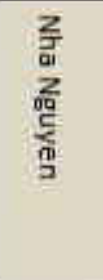 & 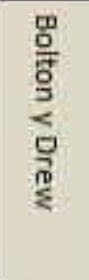 & 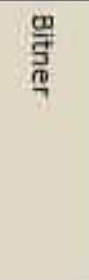 & 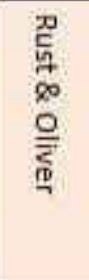 & 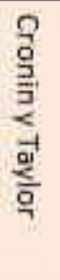 & 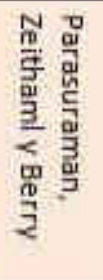 & $\begin{array}{l}\frac{9}{3} \\
\frac{7}{4} \\
\frac{a}{3} \\
0 \\
0 \\
0 \\
\vdots \\
0 \\
0 \\
0\end{array}$ & 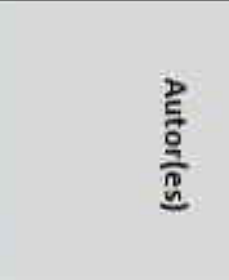 & & \\
\hline 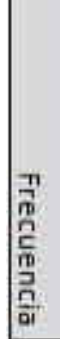 & 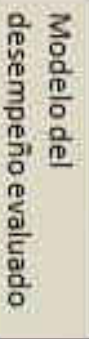 & 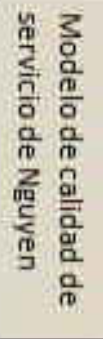 & 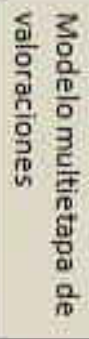 & 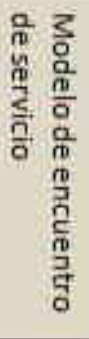 & 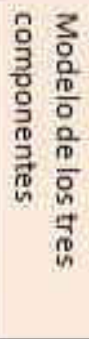 & $\begin{array}{l}\text { 品 } \\
\text { 令 } \\
\text { 留 } \\
\text { n }\end{array}$ & $\begin{array}{l}\text { 盗 } \\
\text { 令 } \\
\text { 号 } \\
\text { ’ }\end{array}$ & 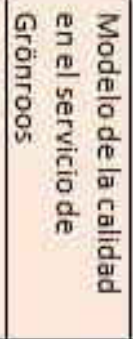 & $\begin{array}{l}\frac{3}{0} \\
\frac{0}{0}\end{array}$ & & \\
\hline or & s & & s & $s$ & $s$ & & $<$ & s & Expectativas & 흠 & \\
\hline$\infty$ & $<$ & $<$ & $<$ & $<$ & $<$ & $<$ & $\varsigma$ & $<$ & Percepciones & g: & \\
\hline n & & & & $<$ & & & & $<$ & Marketing & & \\
\hline$\omega$ & & & $<$ & $<$ & & & & $\leqslant$ & Precios & & \\
\hline$\omega$ & & & $\varepsilon$ & & $<$ & & & $<$ & Experiencias pr & vias & \\
\hline N & & $<$ & & & & & & $<$ & Tradiciones & & \\
\hline N & & $<$ & & & & & & $s$ & Ideologias & $\overline{3}$ & \\
\hline$N$ & & $<$ & & & & & & $<$ & $\begin{array}{c}\text { Comunicación } \\
\text { bocatboca }\end{array}$ & 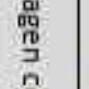 & \\
\hline$\omega$ & & $<$ & & & 5 & & & $<$ & $\begin{array}{c}\text { Cultura } \\
\text { organizacional } \\
\end{array}$ & 음 & 言 \\
\hline$\omega$ & & $<$ & & & 5 & & & 5 & Filosofia & 部 & हैं \\
\hline$v$ & & $<$ & 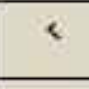 & $<$ & $<$ & $<$ & $<$ & $<$ & $\begin{array}{l}\text { Elementos } \\
\text { tangibles }\end{array}$ & & में \\
\hline a & & $\varsigma$ & 5 & $<$ & 5 & $<$ & $s$ & & Empatia & & 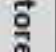 \\
\hline$a$ & & 5 & $<$ & $<$ & $\leqslant$ & 5 & $<$ & & Fiabilidad & $\frac{0}{2}$ & 禺 \\
\hline v & & $\varsigma$ & $\leftarrow$ & $<$ & $<$ & $<$ & $s$ & $<$ & Seguridad & 营器 & \\
\hline$\checkmark$ & & $<$ & \& & $\&$ & $s$ & $<$ & $<$ & $<$ & $\begin{array}{c}\text { Capacidad de } \\
\text { respuesta }\end{array}$ & $\stackrel{0}{3}$ & \\
\hline un & & $<$ & $s$ & $<$ & $<$ & & & $<$ & Caracteristicas & 茜 & \\
\hline in & & $<$ & $<$ & $<$ & $<$ & & & $<$ & Objetivos & o. & \\
\hline
\end{tabular}

Fuente: elaboración propia. 


\section{REFERENCIAS BIBLIOGRÁFICAS}

Ayala, í. M., Camino, J. R., \& Cueva, R. A. (2013). Conducta del consumidor: Estrategias y políticas aplicadas al marketing. Madrid: ESIC.

Baena, J., García, J., Bernal, A., Lara, A., \& Gálvez, P. (2016). "El valor percibido y la satisfacción del cliente en actividades dirigidas virtuales y con técnico en centros de fitness". Revista de Psicología del Deporte 2016, 25(2), 219-227.

Berkowitz, S. (2013). An introduction to structural analysis: the network approach to social research. Toronto: Elsevier.

Bitner, M. (1990). "Evaluating Service Encounters: The Effects of Physical Surroundings and Employee Responses". Journal of Marketing, 54(2), 69-82.

Bitner, M., Booms, B., \& Stanfield, M. (1990). "The service encounter: diagnosing favorable and unfavorable incidents". Journal of Marketing, 54(1), 71-84.

Bolton, R., \& Drew, J. (1991). "A multistage model of customer's assessments of service quality and value". Journal of Consumer Research, 17(4), 375-384.

Camisón, C., Cruz, S., \& González, T. (2006). Gestión de la calidad: conceptos, enfoques, modelos y sistemas. Madrid: Pearson.

Chandrasegaran, S., Ramani, K., Sriram, R., Horváth, I., Bernard, A., Harik, R., y otros. (2013). "The evolution, challenges, and future of knowledge representation in product design systems". Computer-aided design, 45(2), 204-228.

Cronin., J., \& Taylor, S. (1992). "Measuring Service Quality - A Reexamination And Extension". Journal of Marketing, 56(3), 55-68.

Diamandescu, A. (2016). "The main principles of total quality management". Challenges of the Knowledge Society Economic, 6, 672-678.

Eiglier, P., \& Langeard, E. (1987). Servuction : le marketing des services. Paris: McGraw-Hill.

Grönroos, C. (1984). "A service quality model and its marketing implications". European journal of marketing, 18(4), 36-44.

Hoffman, D., \& Bateson, J. (2012). Marketing de servicios: conceptos, estrategias y casos. México: Cengage Learning.

Kotler, P., \& Keller, K. (2012). Dirección de marketing. México: Pearson.

Mercado, S. (2008). Mercadotecnia de servicios. México: PAC.

Moliner, B., Gil, I., \& Ruíz, M. (2014). "Determinantes de la lealtad de empresas turísticas según la heterogeneidad de los segmentos". Papers de Turisme, 55(1), 1-23.

Nguyen, N. (1991). "Un modèle explicatif de l'évaluation de la qualité d'un service : une étude empirique". Recherche et Applications en Marketing, 6(2), 83-98. 
Oliver, R. (1977). "Effect of Expectation and Disconfirmation on Postexposure Product Evaluations: An Alternative Interpretation". Journal of Applied Psychology, 62(4), 480-486.

Oliver, R. (1981). "Measurement and Evaluation of Satisfaction Processes in Retail Settings". Journal of Retailing, 57, 25-48.

Parasuraman, A., Zeithaml, V., \& Berry, L. (1985)."A Conceptual Model of Service Quality and its Implications for Future Research". Journal of Marketing, 41-50.

Parasuraman, A., Zeithaml, V., \& Berry, L. (1988). "Communication and Control Processes in the Delivery of Service Quality". Journal of Marketing, 52(2), 35-48.

Pérez, M. (2014). Control de calidad: Técnicas y herramientas. Madrid: RC Libros.

Rust, R., \& Oliver, R. (1994). Service quality: new directions in theory and practice. Thousand Oaks: Sage.

Teas, K. (1993). "Expectations, Performance Evaluation, and Consumers' Perceptions of Quality". Journal of Marketing, 57(4), 18-34.

Tschohl, J. (2011). Servicio al cliente (Octava ed.). Estados Unidos: Service Quality Institute Latin America. 